\title{
Specific Targeted Integration of Kanamycin Resistance-Associated Nonselectable DNA in the Genome of the Yeast Saccharomyces cerevisiae
}

\author{
Sanjeev K. Waghmare, \\ Valentina Caputo, \\ Slobodanka Radovic, and \\ Carlo V. Bruschi \\ International Centre for Genetic \\ Engineering and Biotechnology, \\ Trieste, Italy
}

\begin{abstract}
Sophisticated genome manipulation requires the possibility to modify any intergenic or intragenic DNA sequence at will, without leaving large amounts of undesired vector DNA at the site of alteration. To this end, a series of vectors was developed from a previous gene knockout plasmid system to integrate nonselectable foreign DNA at any desired genomic location in yeast, with a minimum amount of residual plasmid DNA. These vectors have two mutated Flp recognition targets (FRT) sequences flanking the KanMX4 gene and multiple sites for subcloning the DNA fragment to be integrated. The selectable marker can be recycled by Flp site-specific excision between the identical FRTs, thereby allowing the integration of further DNA fragments. With this system, the NLS-tetR-GFP and DsRed genes were successfully integrated at the thr1 locus, and the RVB1 gene was tagged at the $C$ terminus with the V5-epitope-6-histidine tag. This plasmid system provides for a new molecular tool to integrate any DNA fragment at any genome location in $\left[\mathrm{Cir}^{+}\right]$yeast strains. Moreover, the system can be extrapolated to other eukaryotic cells in which the FLP/FRT system functions efficiently.
\end{abstract}

\section{INTRODUCTION}

In the yeast Saccharomyces cerevisi$a e$, the 2- $\mu \mathrm{m}$ FLP encodes a site-specific recombinase acting on two specific sites, termed the Flp recognition target $(F R T)$, which are located at the center of the inverted repeats $(1,2)$. It had been shown that the Flp protein interacts specifically with a 50-bp DNA sequence that includes three 13-bp repeats; the third of which is inverted with respect to the other two and separated from them by an 8-bp core sequence $(3,4)$. Mutation in the $F R T$ core sequence prevents recombination; however, identical mutations are well tolerated and do not affect site-specific recombination (5-7). Flp can also mediate intermolecular recombination between the two FRTs located on different plasmids $(8,9)$, and it is known that DNA heterology between the FRT core region of the endogenous $2 \mu \mathrm{m}$ and of a foreign plasmid does not permit sitespecific recombination between them. The FLP/FRT system carrying different mutations in the FRT core region has been employed for the construction of recyclable vectors for multiple gene disruption in yeast $(6,10)$. Within the recyclable marker methodology, several other systems are available to date for gene disruption and replacement (11-14) as well as for epitope tagging of chromosomal genes $(15,16)$, but none is capable of integrating at a specific locus any desired DNA sequence that has no directly detectable phenotype. The FLP/FRT system has recently been improved to implement a new, advanced strategy for in vivo genomic
DNA alterations. The new system, called specific targeted integration of kanamycin resistance-associated nonselectable DNA (STIK) allows the integration at any genomic location of DNA sequences that express no directly selectable phenotype, such as spacers, tags, nuclear localization signal (NLS) sequence, and any intergenic or otherwise heterologous sequence. The STIK system accomplishes this task by exploiting the temporary integration of the recyclable, positively selectable KanMX4 marker. To demonstrate the usefulness of this system, the two new plasmids pGKGE and pXKXE were used to perform gene disruption and replacement of the THR1 gene with the NLS-tetR-GFP and DsRed fluorescent markers, respectively, while the new plasmid pHKHE was used to tag the RVB1 gene. After these alterations, the selectable marker could be excised, and thus, similarly, other DNA sequences could be integrated in the same strain.

\section{MATERIALS AND METHODS}

\section{Strains, Media, and Culture Conditions}

The $S$. cerevisiae strain used in this study is the disomic strain for chromosome VIII, Z140-51D: a (thr1/+,CUP1 /+,arg4-2/+,arg4-17/+) his5 ade2 trp1 trp5 leul $\left[\mathrm{Cir}^{+}\right]$, and its derivatives, in which one or both of the thrl alleles were replaced with the fluorescent marker gene GFP: Z140-51G, a (thr1/ THR1::FRTG-NLS-tetR-GFP,CUP1/+, $\arg 4-2 /+, \arg 4-17 /+)$ his5 ade2 trp1 
Table 1. Primers Used in This Work $\left(5^{\prime} \rightarrow 3^{\prime}\right)$

\begin{tabular}{|c|c|}
\hline $\mathrm{THR} 1 \Delta \mathrm{F} 1:$ & $\begin{array}{l}\text { ATGGTTCGTGCCTTCAAAATTAAAGTTCCAGCTTCTTCCGAA } \\
\text { AAATAGGCGTATCACGAG }\end{array}$ \\
\hline THR1 $\Delta R 1:$ & $\begin{array}{l}\text { GCTGTTCGACGCTAGCACCATCGTATGGCAGGCTCAGTAGTC } \\
\text { GATGATAAGCTGTCAAAC }\end{array}$ \\
\hline THR1 $\Delta R 2:$ & $\begin{array}{l}\text { CGAACCTGTTGATAATTTCTTGAGAGATTTCTTCGAATTCTTA } \\
\text { ACTGTGCCCTCCATGG }\end{array}$ \\
\hline THR1F1: & GAGTCATCATCTCGAAAAG \\
\hline THR1R1: & TTAGCATCAGAACGCAATGG \\
\hline $1-\mathrm{V} 5 \mathrm{~F}:$ & $\begin{array}{l}\text { AAGGTCAACAAAGATTTTAGAAACTTCCGCAAATTATTTG } \\
\text { AAGGGCGAGCTTCGAGGTCAC }\end{array}$ \\
\hline 1-V5R: & $\begin{array}{l}\text { TATTTTTATTTATGAAATGTGCTTTAGGCTTTCTTCACTGTCG } \\
\text { ATGATAAGCTGTCAAAC }\end{array}$ \\
\hline RVB1F2: & CAAGATGTTACCTTGCACGA \\
\hline RVB1R2: & CAAGTAATAGCAGCAACAAC \\
\hline K1: & CAATCGATAGATTGTCGCAC \\
\hline K2: & TTATGCCTCTTCCGACCATC \\
\hline Polylinker: & TACGTACGTACGCCGCGGCCGCGAATTC \\
\hline
\end{tabular}

trp5 leu1 $\left[\mathrm{Cir}^{+}\right]$, and GFP plus DsRed: Z140-51GR, a (thr1::RFP-FRTH/ THR1::FRTG-NLS-tetR-GFP,CUP1/+, $\arg 4-2 /+, \arg 4-17 /+)$ his5 ade2 trpl trp5 leu1 $\left[\mathrm{cir}^{+}\right]$, respectively. Diploid strain DUPOT-SL: gal2/+,leu2/leu2 $\therefore F R T X$,arg10/+,ade2/+,ura3-52/ura3$5\left[\mathrm{Cir}^{+}\right]$and haploid strain YPH250: a ade2 leu2 lys 2 his3 trp1 ura3 $\left[\mathrm{cir}^{+}\right]$ were also used. Yeast peptone dextrose (YPD) and kanamycin-containing media were prepared as described (17). The yeast transformation protocol was used as described in the European Functional Analysis Network (EUROFAN) program manual (http://www. mips.biochem.mpg.de/proj/eurofan/eurofan $1 / \mathrm{b} 0 /$ home requisites/guideline/ exp-transformation.html). E. coli strain $\mathrm{DH} 5 \alpha$ was used for plasmid propagation. E. coli cultures were grown in LB broth supplemented ampicillin as previously described (17).

\section{Plasmids}

The YIplac128 vector, harboring the NLS-tetR-GFP chimeric DNA fragment under the yeast URA3 promoter and followed by the $A D H 1$ terminator, was obtained from Kim Nasmyth's laboratory (IMP, Vienna, Austria). The
Living Colors ${ }^{\circledR}$ pDsRed1-N1 vector was obtained from BD Biosciences Clontech (Palo Alto, CA, USA). In the DsRed gene construct, the cytomegalovirus $(C M V)$ promoter was replaced with the yeast $L E U 2$ promoter. Plasmid pTOPO-RUVB containing the Bacillus subtilis RuvB gene fused in frame with the V5His6x epitope was constructed in our laboratory using the pYES2.1 TOPO TA ${ }^{\mathrm{TM}}$ Cloning Kit (Invitrogen, Carlsbad, CA, USA). Plasmids pGKG, pXKX, and pHKH, bearing different mutations in the central core region of the FRT sequence and pWKW, having wild-type $F R T$ s, were constructed in our laboratory (6). From these plasmids, the new series of vectors was obtained as described in the Results section, an example of which is shown in Figure 1A.

\section{Primers}

Table 1 lists the primers used in this study, and their details are as follows. THR1 $\Delta \mathrm{F} 1$ : chimeric forward primer, having a 40-bp homologous sequence from the ATG start codon of the THR1 gene and $20 \mathrm{bp}$ homologous upstream of the $5^{\prime} F R T$; THR1 $\triangle \mathrm{R} 1$ : chimeric reverse primer, having $40 \mathrm{bp}$ homologous 


\section{Research Report}

to the downstream sequence of THR 1 locus and $20 \mathrm{bp}$ homologous downstream of the $3^{\prime} F R T$; THR1 $\triangle \mathrm{R} 2$ : chimeric reverse primer, having $40 \mathrm{bp}$ homologous to the sequence of THRI locus, further upstream of the THR1 $\Delta \mathrm{R} 1$ primer, and $20 \mathrm{bp}$ homologous downstream of the FRT; THR1F1: forward primer homologous to $100 \mathrm{bp}$ upstream of the ATG of the THR1 gene; THR1R1: reverse primer homologous to $300 \mathrm{bp}$ downstream of the ATG of the THR1 gene; 1-V5 F: chimeric forward primer, having $40 \mathrm{bp}$ homologous to the sequence immediately upstream of the RVB1 STOP codon and 20 bp homologous to the sequence upstream of the V5 epitope in pH-RuvBV5H6; 1-V5 R: chimeric reverse primer, having $40 \mathrm{bp}$ homologous to the sequence immediately downstream of the RVB1 STOP codon and $20 \mathrm{bp}$ homologous to the sequence downstream of the FRT; RVB1F2: forward primer homologous to the sequence $533 \mathrm{bp}$ upstream of the STOP codon of the RVB1 gene; RVB1R2: reverse primer homologous to the sequence $364 \mathrm{bp}$ downstream of the STOP codon of the RVB1 gene; K1: reverse primer homologous to a 500-bp sequence downstream of the ATG of the KanMX4 gene; and K2: forward primer homologous to the sequence 898 bp upstream of the STOP codon of the KanMX4 gene.

\section{Standard Molecular Biol- ogy Techniques}

Plasmid DNA was extracted from $E$. coli using the Wizard ${ }^{\circledR}$ Plus mini-preparation kit (Promega, Madison, WI, USA). Restriction enzymes and the DNA polymerase I Klenow fragment were obtained from New England Biolabs (Beverly, MA, USA) and used according to the manufacturer's recommendations. Restriction fragments were separated by gel electrophoresis and purified using the QIAquick ${ }^{\circledR}$ gel extraction kit (Qiagen, Valencia, CA, USA), as specified by the manufacturer.

\section{Fluorescent Microscopy}

The yeast strain was grown on G418 plates, and the cells were harvested and washed once with water. Approximately $10^{7}$ cells were mounted on a glass slide and air-dried. Subsequently, $5 \mu \mathrm{L}$ mounting medium (Vector Laboratories, Burlingame, CA, USA) were added over the dried culture. The green fluorescence was visualized using an Axiovert ${ }^{\circledR} 100 \mathrm{M}$ confocal microscope (Carl Zeiss Jena, Jena, Germany).

\section{PCR Analysis}

Yeast colony PCR. Yeast colonies picked from the plate were suspended in $50 \mu \mathrm{L}$ sterile water and incubated with $5 \mu \mathrm{L}$ Lyticase $^{\mathrm{TM}}$ (Sigma, St. Louis, $\mathrm{MO}, \mathrm{USA})$ at $40 \mathrm{U} / \mu \mathrm{L}$ for $15 \mathrm{~min}$ at room temperature. The suspension was centrifuged at $400 \times g$ for $1 \mathrm{~min}$, harvested, and the supernatant was discarded. Cells were denatured at $100^{\circ} \mathrm{C}$ for $10 \mathrm{~min}$ and placed on ice for $5 \mathrm{~min}$. The pellet was then suspended in $40 \mu \mathrm{L}$ sterile water, and $10 \mu \mathrm{L}$ were used as a template for PCR. The reaction was performed following the standard EUROFAN B0 Program protocol. PCR for the amplification of replacement min at $94^{\circ} \mathrm{C}$, followed by 20 cycles at $94^{\circ} \mathrm{C}$ for $30 \mathrm{~s}, 54^{\circ} \mathrm{C}$ for $30 \mathrm{~s}$, and $68^{\circ} \mathrm{C}$ for $4 \mathrm{~min}$. Subsequently, there were 10 cycles at $94^{\circ} \mathrm{C}$ for $30 \mathrm{~s}, 59^{\circ} \mathrm{C}$ for $30 \mathrm{~s}$, and $68^{\circ} \mathrm{C}$ for $4 \mathrm{~min}$, followed by one cycle of $68^{\circ} \mathrm{C}$ for $5 \mathrm{~min}$. A mixture of Taq DNA polymerase and Pfu DNA polymerase (Promega) in a ratio of $1: 1$ was used for the amplification of the replacement cassettes. After amplification, $10 \mu \mathrm{L}$ of the samples were run on a standard $1.2 \%$ agarose gel for size analysis.

\section{Subcloning the NLS-tetR-GFP, DsRed, and RUVB-V5His6x DNA Fragment in the pGKGE, pXKXE, and pHKHE Vectors}

To subclone NLS-tetR-GFP in the pGKGE vector, it was cut with the SnaBI restriction enzyme to create blunt ends. The 2.2-kb region bearing the NLS-tetR-GFP fragment was excised from the vector YIplac128 by cassettes consisted of one cycle of 2

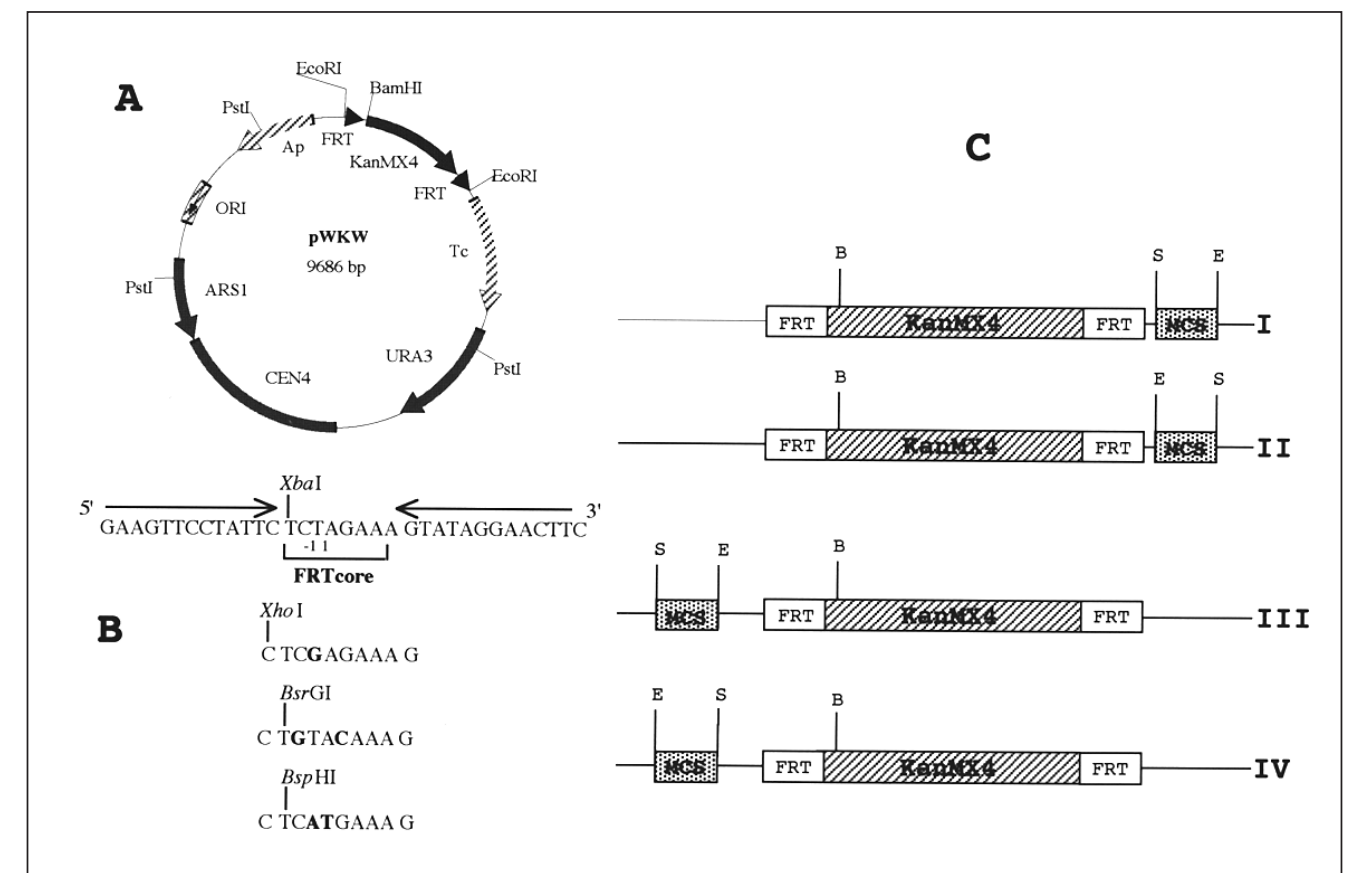

Figure 1. Schematic representation of the wild-type $\mathbf{p W K W}$ vector. Panel A shows the major plasmid elements and restriction sites. The different sequences of the $F R T$ core present on the various vectors are indicated in panel $\mathrm{B}$. The wild-type $F R T$ sequence with restriction site $X b a$ I, the $F R T$ sequence with the mutation generating the $X h o$ s site that generated the $B s r \mathrm{GI}$ site, which generated the $B s p \mathrm{HI}$ site, are present on the plasmids pWKW, pXKX, pGKG, and pHKH, respectively. In this vector, the MCS has been cloned in both orientations, in either EcoRI restriction site, giving rise to the four types of vectors (I, pHKGE; II, pXKXE; III, pHKHE; and IV, pWKWE) represented in panel C. 


\section{Research Report}

double digestion with restriction enzymes EcoRI and BamHI. The recessed $5^{\prime}$ ends of this fragment were gap-filled using DNA polymerase I Klenow fragment. Lastly, this $2.2-\mathrm{kb}$ fragment was further subcloned into the pGKGE vector (Figure 1C, I). Similarly, the DsRed fragment was subcloned into the pXKXE vector (Figure 1C, II).

To subclone RuvB-V5His6x in the pHKHE vector, pTOPO-RuvB was cut by double digestion with $P v u I I$ and $X b a \mathrm{I}$ restriction enzymes. The recessed $5^{\prime}$ end of this fragment was gap-filled using the DNA polymerase I Klenow fragment. Finally, the $1.5-\mathrm{kb}$ DNA fragment was subcloned into pHKHE SnaBI site (Figure 1C, III), which gave rise to the plasmid $\mathrm{pH}-\mathrm{RuvBV} 5 \mathrm{His} 6 \mathrm{x}$.

\section{Immunochemical Techniques}

The V5 epitope-tagged protein was detected using the mouse monoclonal
Anti-V5 Antibody (Invitrogen). Whole protein extracts were prepared according to the protocol available (http://www. pmci.unimelb.edu.au/core facilites/ manual $/ \mathrm{mb} 460$.asp). Western blot analysis was performed as previously described (17). The proteins were visualized on the blot membrane after Western blot hybridization using a stain with a solution of $0.1 \%$ Ponceau-S Red stain and $1 \%$ acetic acid for $1 \mathrm{~min}$.

\section{RESULTS}

\section{Construction of New Recyclable \\ STIK Vectors for Genomic Integration of Nonselectable DNA Fragments}

A series of vectors carrying the selectable marker KanMX4 flanked by different FRT sequences such as pWKW, pGKG, pHKH, and pXKX were previ-

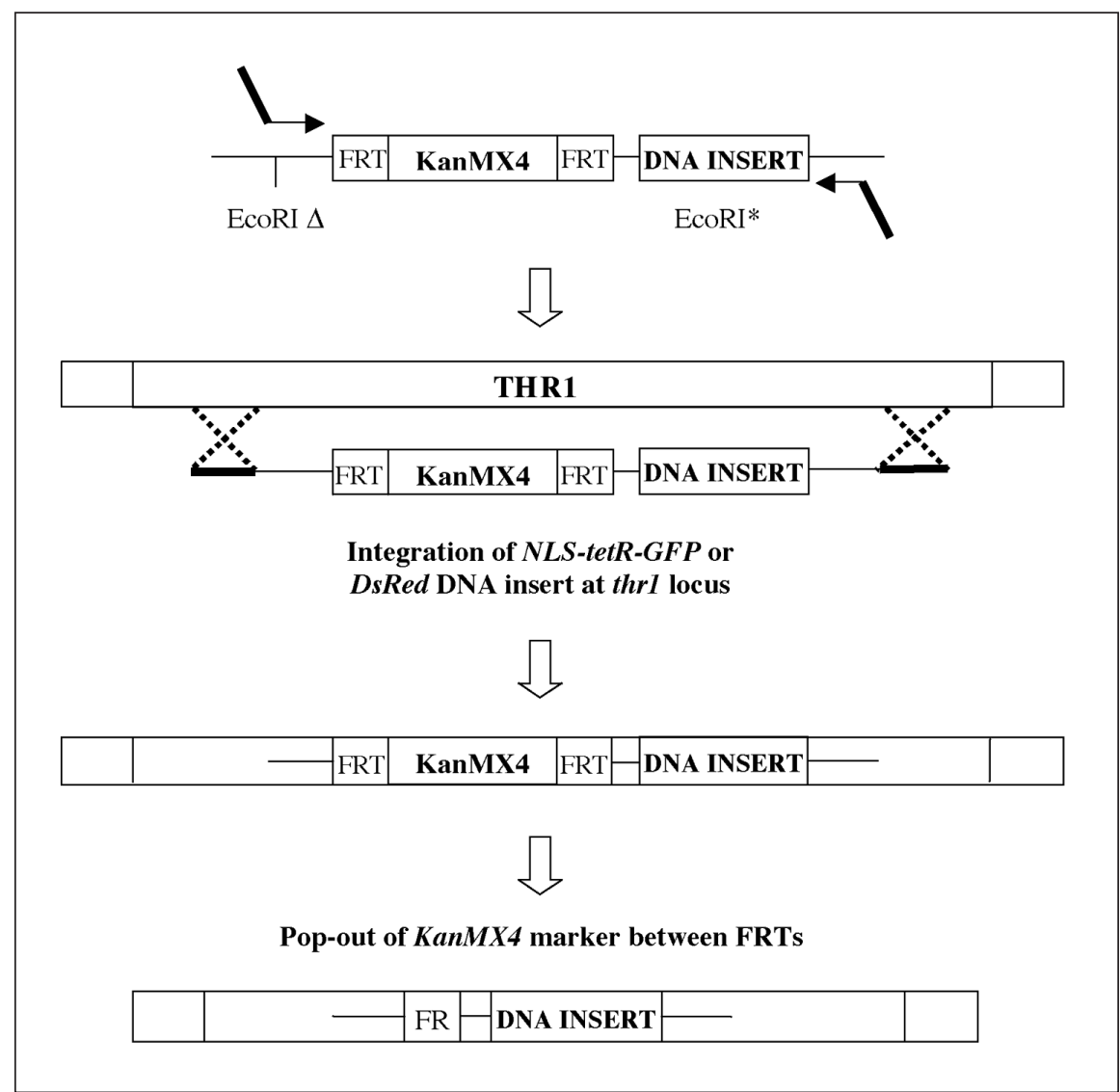

Figure 2. Schematic representation of the STIK-based integration of an NLS-tetR-GFP or DsRed fluorescent marker at the thrl locus on chromosome VIII. After the integration of the fluorescent marker and pop-out of KanMX4, the second fluorescent marker has been integrated in the same way into the second allele. EcoRI $\Delta$ and EcoRI*, remaining and deleted restriction site, respectively. ously constructed in our laboratory (Figure 1A) $(6,10)$. The vector pWKW has wild-type $F R T$ sequences, whereas the other three vectors have different mutations in the FRT core region. Figure 1B shows the sequences of the wild-type and mutated FRT. These vectors were further modified by deleting one of the two EcoRI sites flanking the FRTs and subcloning a multiple cloning site (MCS) (SnaB1, Spl1, SacII, NotI, and EcoRI) at the remaining EcoRI site. The position and orientation of the MCS were determined by DNA sequencing (CRIBI, University of Padova) (Figure 1C). These modified vectors were designated as pGKGE, pXKXE, pHKHE, and $\mathrm{pWKWE}$, according to the core $F R T$ sequence present and the EcoRI restriction site that was used for cloning (Figure 1C, I-IV, respectively). The MCS enables the subcloning of the desired sequence in the plasmid to be used as a template for the PCR amplification of the integration cassette. After the integration of the cassette, Flp-induced sitespecific recombination occurs only between identical FRT sequences, thus allowing the excision of the selectable marker, while leaving one FRT scar sequence integrated in the DNA, together with the sequence of interest (Figure 2).

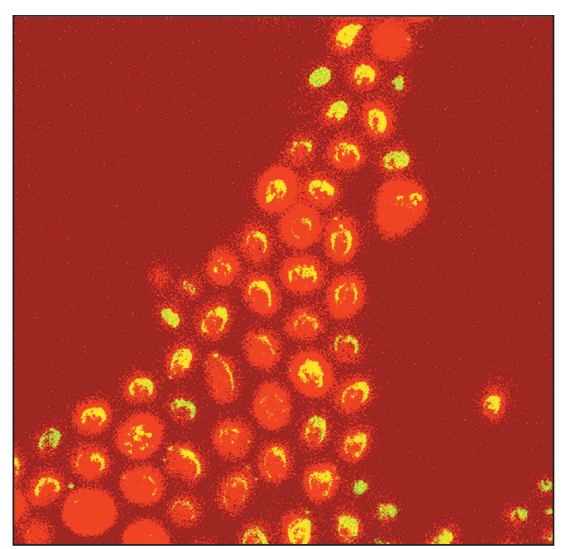

Figure 3. Confocal fluorescence microscopy of disomic cells $(1 \mathrm{n}+1)$ of the yeast $S$. cerevisiae harboring $N L S$-tetR-GFP and DsRed fluorescent marker genes each integrated at the $t h r 1$ locus on the two copies of chromosomes VIII. The green fluorescence is visible within the nucleus of the cells, while the red is present in the cytoplasm. Cells that have both disomic chromosomes exhibit both red and green fluorescence. Some cells have lost either the green or red fluorescence, indicating the loss of the related copy of the chromosome. 


\section{Integration of the NLS-tetR-GFP and DsRed Sequence at the THR1 Loci}

To demonstrate that our system could be used for repeated targeted integration, we replaced both threonine (thrl) loci on chromosome VIII in $S$. cerevisiae strain Z140-51D disomic for that chromosome. Each THRI and thr 1 threonine allele was subsequently replaced with NLS-tetR-GFP and DsRed constructs, respectively. The $t e t R$ gene fused in frame with the simian virus 40 (SV40) $N L S$ sequence at its $5^{\prime}$ end and GFP at its $3^{\prime}$ end was subcloned into the pGKGE plasmid (Figure 1C, I). This plasmid was then used as a template for the amplification of the replacement cassette using primers THR $1 \Delta \mathrm{F} 1$ and $\mathrm{THR} 1 \Delta \mathrm{R} 1$. Both the forward and reverse $\mathrm{THR} 1 \Delta \mathrm{F} 1$ and $\mathrm{THR} 1 \Delta \mathrm{R} 1$ chimeric primers $(60 \mathrm{bp})$ were designed in such a way that $40 \mathrm{bp}$ are homologous to the sequences upstream and downstream, respectively, within THR 1 . The remaining $20 \mathrm{bp}$ are homologous to the sequence of the vector upstream of the $5^{\prime} F R T$ and to the subcloned $N L S$-tetR-GFP fragment, respectively (Figure 2). Yeast strain ZI40-51D was transformed with the PCR-amplified, linear 3.6-kb NLS-tetR-GFP cassette, and the transformants were selected on YPD plates supplemented with geneticin (G418). Eventually, the THR1 gene was substituted with the $N L S$ tetR-GFP and two FRTs flanking the KanMX4 gene. To confirm that the integration event occurred at the THRI locus, geneticin-resistant transformants were further analyzed by yeast colony PCR using primers THRF1, THRR1, and K1 (data not shown). Moreover, the new strain, designated as $\mathrm{Z} 140-51 \mathrm{G}$, harboring the substitution of THR 1 with the NLS-tetR-GFP cassette, fails to grow on minimal medium without threonine, as expected. The KanMX4 marker was excised spontaneously by growing the G418resistant, Z140-51G strain in the nonselective liquid medium (YPD) for several generations to relieve the selective pressure for the marker. Approximately one hundred cells per plate were plated onto solid YPD. After incubation, the colonies obtained were further replica-plated onto YPD containing G418 to screen for G418sensitive colonies. The frequency of the G418-sensitive colonies was found to be of the order of $10^{-3}$, which is similar to that reported for the former set of knockout plasmid vectors (6). The loss of the marker was further confirmed by yeast colony PCR analysis using primers THR1F1, THRR1, and $\mathrm{K} 1$. At this stage, the remaining thrl locus was similarly replaced with the DsRed sequence encoding the red protein. This was initially subcloned into the pXKXE vector (Figure 1C, II) that served as a template for the PCR amplification of the DsRed replacement cassette using primers $\mathrm{THR} 1 \Delta \mathrm{F} 1$ and THR1 $\Delta \mathrm{R} 2$. The cassette was then integrated at the remaining threonine locus, which created a new G418-resistant strain Z140-51GR. The integration event was again confirmed by PCR analysis using primers THR1F1, THRR1, and K1 (data not shown).

The green and red fluorescence thus expressed by the integrated NLS-tetRGFP and DsRed genes was visualized using confocal microscopy (Figure 3). From the picture, one can see that the fluorescence is present in almost all of the cells. The green fluorescence is localized within the nucleus due to the presence of the SV40 virus NLS sequence in the tetR-GFP construct, while the DsRed protein stains the entire cell due to its absence. Thus, the $N L S$-tetR-GFP and DsRed genes were successfully integrated at both thr 1 loci.

\section{Integration of the PCR-Tagging Cassette}

To demonstrate the versatility of the STIK system for tagging chromosomal genes, we subcloned a DNA fragment encoding the V5His6x epitope into plasmid pHKHE (Figure 1C, III) to create plasmid $\mathrm{pH}-\mathrm{V} 5 \mathrm{His} 6 \mathrm{x}$, which was used as a template for the construction of the tagging cassette. The 65-bp forward $(1-\mathrm{V} 5 \mathrm{~F})$ and reverse (1-V5R) chimeric primers required for the construction of the RVB1 locus tagging cassette are reported in Table 1 , and their position relative to target genomic sequence is shown in Figure 4.

Both haploid YPH250 and diploid DUPOT-SL cells were transformed with the $1.75-\mathrm{kb}$ PCR amplified linear tagging cassette, and transformants were selected on YPD plates supplemented with geneticin (G418). Cassette integration was confirmed by yeast colony PCR analysis using primers RVB1F2, RVB1R2, and K2 (data not shown). No further excision of the KanMX4 marker was applied in this case. Whole cell proteins were visualized by staining the membrane with Ponceau-S Red stain (Figure 5, left panel). The transformants were assayed for correct expression of the desired Rvb1p-tagged variant by Western blot analysis (Figure 5, right panel). Figure 5 shows that the insertion of the DNA sequence tag was successful and did not alter the expression or the structure of the Rvb1 protein.

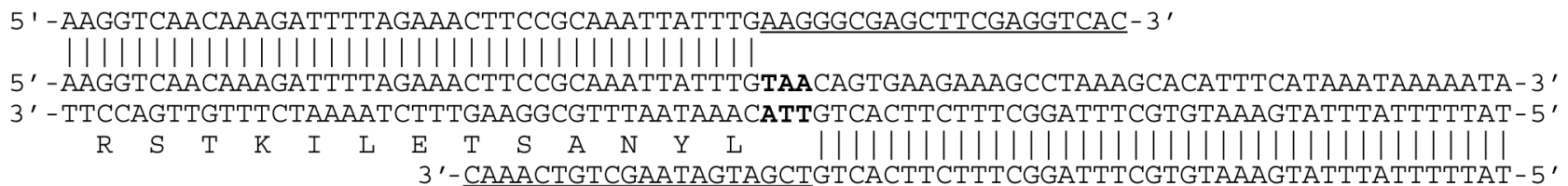

Figure 4. Position of the chimeric primers 1-V5F and 1-V5R used for the in-frame cloning of the V5His6x epitope at the $3^{\prime}$ end of $R V B 1$. Forty base pairs of the forward and reverse primers (vertical lines) are homologous to the RVBI ORF sequence upstream, and within its terminator downstream, of the stop TAA codon (in bold), respectively. Additional 20 bases complementary to the subcloned V5His6x DNA fragment and to the sequence outside the FRTs (corresponding to the vector) are underlined. 


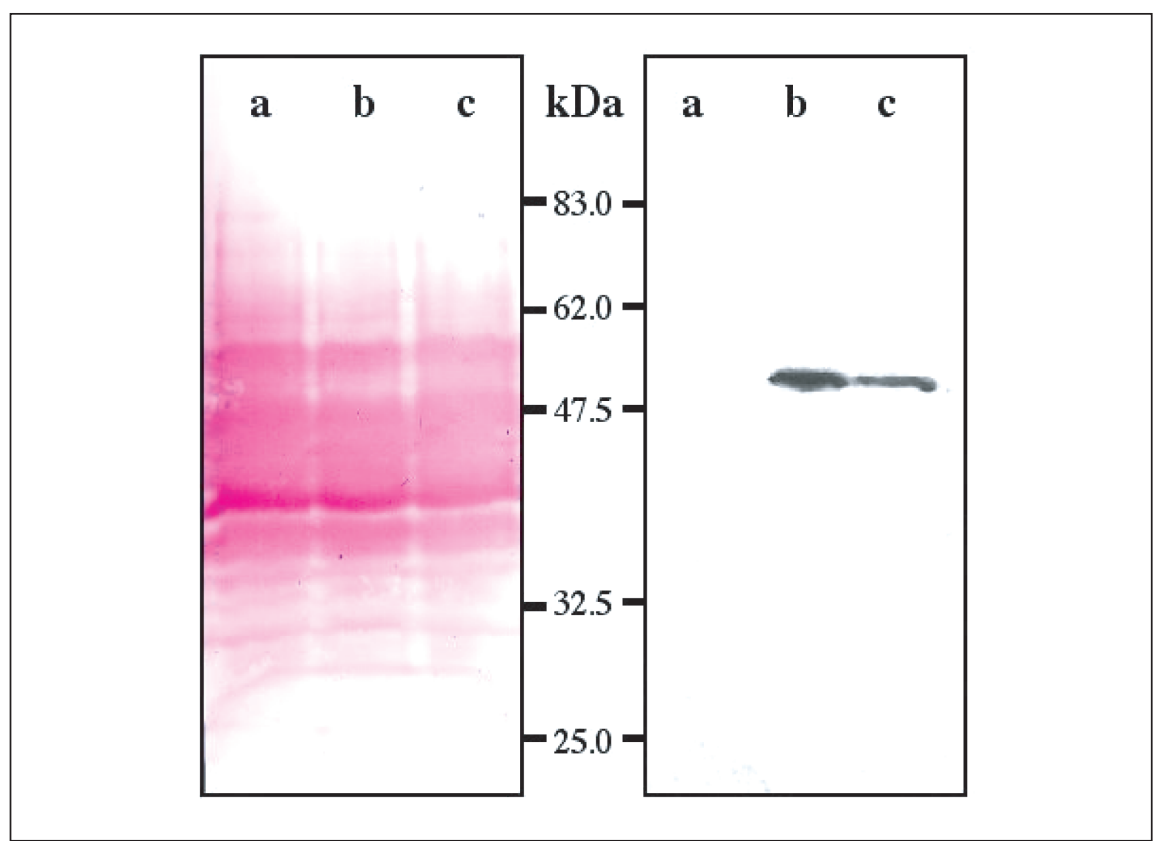

Figure 5. Whole cell proteins visualized by Ponceau-S Red staining (left panel) and Western blot analysis (right panel) from yeast strains: lane a, wild-type; lane b, RVB1-tagged YPH250; and lane c, $R V B 1$-tagged DUPOT-SL.

\section{DISCUSSION}

The objective of this work was to construct a series of plasmid vectors that could be used to integrate any DNA sequence of interest, including those nonselectable phenotypically, to a desired coding or noncoding genomic target location using the FLP/FRT-mediated recombination system and the KanMX4 as a selectable marker in $S$. cerevisiae. A series of PCR-template vectors bearing different mutations in the core region of the FRTs was previously reported from our laboratory $(6,10)$. These vectors enable one to perform multiple gene disruption and knockout using a recyclable selectable marker. In our new STIK system, the PCR-amplified FRT-KanMX4-FRT DNA fragment directs the integration to the genomic location of homology, allows its own positive selection, and then it is lost by FLP/FRT-mediated site-specific excision. This leaves the DNA sequence of interest integrated at the selected chromosomal location, together with a 54-bp DNA "scar" consisting of $20 \mathrm{bp}$ homologous to the plasmid template, which can be either $5^{\prime}$ TCGATGATAAGCTGTCAAAC-3' or 5'-AAAAATAGGCGTATCACGAG-
3', depending on the EcoRI site that was used for polylinker insertion and $34 \mathrm{bp}$ of the mutated FRT employed (6). Recombination between wild-type and mutated scars, as well as between different FRT scars is not favored; therefore, their presence is not inducing any genomic instability due to internal recombination. The most salient feature of our work is that our newly constructed series of PCR template vectors, pGKGE, pHKHE, pXKXE, and pWKWE, overcomes the limitation laid down by the impossibility of performing the targeted integration of DNA fragments for which there is no direct selection possible. Therefore, this system provides a broad horizon of possibilities to integrate any nonhomologous DNA sequence at the desired chromosomal location, without leaving relevant exogenous vector sequences that could interfere with the experiment. All the above vectors can be used in $\left[\mathrm{Cir}^{+}\right]$ strains; however, pWKWE can only be used in $\left[\mathrm{cir}^{\circ}\right]$ strains due to the presence of wild-type FRTs in this vector that could recombine with the FRTs of the endogenous $2-\mu \mathrm{m}$ circle. The extrapolation of this system to $\left[\mathrm{cir}^{\circ}\right]$ strains involves the presence of the endogenous FLP site-specific recombinase 
gene in the vectors themselves. The existing plasmids, pGFKF, pHFKH, pXFKX from our previous work (6), carrying the endogenous FLP gene, can be used as substrate for the construction of additional vectors to be employed in $\left[\mathrm{cir}^{\circ}\right]$ strains.

To validate our work, the NLS-tetR$G F P$ and DsRed DNA fragments were subcloned in the pGKGE and pXKXE vectors, respectively, and the entire region was PCR amplified along with the two FRTs and KanMX4. These cassettes were integrated in the ZI40-51D strain at the thrl locus on chromosome VIII, and the phenotypic expression of the fluorescent markers was observed by confocal microscopy (Figure 3). Moreover, to demonstrate the usefulness of this system for protein tagging, we constructed a module that contains the V5 epitope in combination with a 6 His-tag. This module allows for the expression of tagged proteins under their own regulatory elements and, subsequently, their immediate use for a large set of biochemical and cell biology tests (14). We tagged the essential RVB1 gene in haploid and diploid genetic background using the YPH250 and DUPOT-SL strain, respectively. Both strains correctly expressed the C-terminal tagged Rvb1 protein, as demonstrated by Western blot analysis (Figure 5), although in this experiment, the selectable KanMX4 marker was not popped out. This also confirms that the presence of the $3^{\prime}$ FRT in the construct can act as an effective transcription terminator as previously reported (7) because the original terminator sequence was disrupted in the tagging process.

Our STIK plasmid system can be used for the construction of multiple deletions, replacement of endogenous regulatory elements, and tagging of gene products with high efficiency, using the common KanMX4 as a recyclable selectable marker. Furthermore, this plasmid system can be easily extrapolated to other eukaryotic cells like Schizosaccharomyces pombe, Drosophila melanogaster, maize, Xenopus laevis embryos, and human cells, in which the FLP/FRT site-specific recombination has been demonstrated to function effectively $(16,18-21)$. In these cases, an inducible FLP recombinase gene should be cotransfected together with the new vector.
This new STIK system will greatly benefit, in particular, those experiments of cell biology in which fewer marker genes are available for DNA sequence integration.

To this end, we have made constructions of the plasmid series containing the GFP variant blue fluorescent protein (BFP) and the herpes simplex virus (HSV) epitope tag. We will also be available to insert other commonly used tags, such as GST, if requested.

\section{ACKNOWLEDGMENTS}

We would like to thank V. Tosato and K. Nasmyth for providing us with the $R u v B$ and the $N L S$-tetR-GFP genes, respectively, and V. Rapisarda for technical help.

\section{REFERENCES}

1.Broach J.R. and F.C. Volkert. 1991. The Molecular and Cellular Biology of Yeast Saccharomyces: Genome Dynamics, Protein Synthesis and Energetics. CSH Laboratory Press, Cold Spring Harbor, NY

2.Senecoff, J.F. and M.M. Cox. 1986. Directionality in FLP protein-promoted site-specific recombination is mediated by DNA-DNA pairing. J. Biol. Chem. 261:7380-7386.

3.Andrews, B.J., G.A. Proteau, L.G. Beatty, and P.D. Sadowski. 1985. The FLP recombinase of the 2 micron circle DNA of yeast: interaction with its target sequences. Cell 40:795-803.

4.Senecoff, J.F., P.J. Rossmeissl, and M.M. Cox. 1988. DNA recognition by the FLP recombinase of the yeast $2 \mu \mathrm{m}$ plasmid. A mutational analysis of the FLP binding site. J. Mol. Biol. 201:405-421.

5.Dixon, J.E. and P.D. Sadowski. 1994. Resolution of immobile chi structures by the FLP recombinase of 2 microns plasmid. J. Mol. Biol. 243:199-207.

6.Storici, F., M. Coglievina, and C.V. Bruschi. 1999. A 2-micron DNA-based marker recycling system for multiple gene disruption in the yeast Saccharomyces cerevisiae. Yeast 15:271-283.

7.Storici, F. and C.V. Bruschi. 2000. Involvement of the inverted repeat of the yeast 2-micron plasmid in Flp site-specific and RAD52dependent homologous recombination. Mol. Gen. Genet. 263:81-89.

8.Bruschi, C.V. and G.A. Howe. 1988. High frequency FLP-independent homologous DNA recombination of $2 \mu \mathrm{m}$ plasmid in the yeast Saccharomyces cerevisiae. Curr. Genet. 14:191-199.

9.Bruschi, C.V. and D.L. Ludwig. 1989. Introduction of nonselectible $2 \mu \mathrm{m}$ plasmid into [cir ${ }^{\circ}$ ] cells of the yeast $S$. cerevisiae by DNA transformation and in vivo site-specific reso- lution. Curr. Genet. 15:83-90.

10.Storici, F. and C.V. Bruschi. 1997. Molecular engineering with the FRT sequence of the yeast 2 micron plasmid: $\left[\mathrm{cir}^{\circ}\right.$ ] segregant enrichment by counterselection for 2 micron site-specific recombination. Gene 195:245255 .

11.Akada, R., I. Hirosawa, M. Kawahata, H Hoshida, and Y. Nishizawa. 2002. Sets of integrating plasmids and gene disruption cassettes containing improved counter-selection markers designed for repeated use in budding yeast. Yeast 19:393-402.

12.Reid, R.J., I. Sunjevaric, M. Keddache, R Rothstein, and M. Kedacche. 2002. Efficient PCR-based gene disruption in Saccharomyces strains using intergenic primers. Yeast 19:319 328.

13.Toh-e, A. 1995. Construction of a marker gene cassette which is repeatedly usable for gene disruption in yeast. Curr. Genet. 27:293297.

14.Wach, A., A. Brachat, R. Pohlmann, and P. Philippsen. 1994. New heterologous modules for classical or PCR-based gene disruptions in Saccharomyces cerevisiae. Yeast 10:17931808

15.De Antoni, A. and D. Gallwitz. 2000. A novel multi-purpose cassette for repeated integrative epitope tagging of genes in Saccharomyces cerevisiae. Gene 246:179-185.

16.Knop, M. and E. Schiebel. 1997. Spc98p and Spc97p of the yeast gamma-tubulin complex mediate binding to the spindle pole body via their interaction with Spc110p. EMBO J. 16:6985-6995.

17.Sambrook, J., E.F. Fritsch, and T. Maniatis. 1989. Molecular Cloning: A Laboratory Manual, 2nd ed., CSH Laboratory Press, Cold Spring Harbor, NY.

18.Golic, M.M., Y.S. Rong, R.B. Petersen, S.L. Lindquist, and K.G. Golic. 1997. FLP-mediated DNA mobilization to specific target sites in Drosophila chromosomes. Nucleic Acids Res. 25:3665-3671.

19.Lyznik, L.A., K.V. Rao, and T.K. Hodges. 1996. FLP-mediated recombination of FRT sites in the maize genome. Nucleic Acids Res. 24:3784-3789.

20.Ng, P., D.T. Cummings, C.M. Evelegh, and F.L. Graham. 2000. Yeast recombinase FLP functions effectively in human cells for construction of adenovirus vectors. BioTechniques 29:524-528.

21.Werdien, D., G. Peiler, and G.U. Ryffel. 2001. FLP and Cre recombinase function in Xenopus embryos. Nucleic Acids Res. 29:E53-53.

Received 26 August 2002; accepted 25 February 2003.

Address correspondence to:

Prof. C.V. Bruschi

Senior Scientist and Head

ICGEB Microbiology Group

Area Science Park, Padriciano-99

Trieste, Italy

e-mail:bruschi@icgeb.org 